\title{
Influence of cultivation conditions on IAA - producing activity of endophytic bacterial strains isolated from morning-glory (IPOMOEA PES-CAPRAE (L.) R.Br.)
}

\author{
Nguyen Van Zhang ${ }^{1}$, Nguyen Thi Thu ${ }^{1}$, Vu Thi Linh ${ }^{1}$, V.V.Pylnev², and M.I. Popchenko ${ }^{2}{ }^{*}$ \\ ${ }^{1}$ National Agricultural University of Vietnam, Hanoi, Vietnam \\ ${ }^{2}$ Russian State Agrarian University - Moscow Agricultural Academy named after K. A. Timiryazev, \\ Moscow, Russia
}

\begin{abstract}
This work presents the experimental study results of the influence of the culture medium on the ability to IAA synthesis of three endophytic strains TH10R, TH11T, and TH13T from roots of Ipomoea pes-caprae. Three investigated strains give the highest IAA concentration after $96 \mathrm{~h}$ of cultivation. A significant increase in IAA biosynthesis was obtained by cultivating the TH10R strain in a medium containing lactose or starch as a carbon source and $\mathrm{NH} 4 \mathrm{Cl}$ or $\mathrm{KNO} 3$ as a nitrogen source. The TH11T strain produces the maximum amount of IAA, using glucose or xylose and $\mathrm{KNO} 3$ or $\mathrm{NH} 4 \mathrm{NO} 3$ as carbon and nitrogen sources, respectively. Sucrose is a suitable carbon source for the TH13T strain; on a sucrose-containing medium, the TH13T strain produces the highest IAA amount. The most active strain is TH10R, identified as Bacillus mycoides and named Bacillus mycoides TH10R.
\end{abstract}

\section{Introduction}

The term "endophyte" is broadly used for fungi, bacteria or other microorganisms that colonize living tissues of a plant without causing obvious pathological symptoms. Being an important integrative part of the phytobiome, endophytes have a complex of economically valuable property, such as nitrogen fixation, mobilization of hard-to-reach mineral elements from the environment (for example, phosphorus, potassium, zinc), detoxification, inhibition of pathogens and pests, inducing resistance to stress abiotic factors and environmental pollution, stimulation of plant growth and enhancement of phytoimmunity [1,2]. Recently, endophytes and rhizosphere bacteria capable of stimulating plant growth (the so-called "PGPR") are increasingly entering the practice of modern agrobiotechnology. Currently, interest in the use of biological products based on microorganisms that can stimulate the growth and development of plants and prevent the spread of infections is increasing in crop production [1]. One of these products is IAA (Indole-3-acetic acid) - the most common natural auxin that affects the most important physiological processes in plants.

\footnotetext{
*Corresponding author: popchenko_m@inbox.ru
} 
Ipomoea pes-caprae (L.) R.Br. plays an important role in coastal ecosystems, participating in the natural protection of the coastline from erosion. The plant has the ability to live in harsh and unstable environmental conditions due to a complex of adaptive properties, among which symbiosis with endophytic bacteria is among most important.

\section{Materials and research methods}

Root samples of Ipomoea pes-caprae were collected from Ngang Beach, Thang Hai Village, Tinh Hai Commune, Tinh Gia District, Thanh Hoa Province. 15 strains of endophytic bacteria were obtained from the roots, including 11 strains actively synthesizing IAA, 12 strains with siderophore-producing ability, and 6 strains with phosphate-dissolving activity. Three strains most actively synthesizing IAA: TH10R, TH11T, and TH13T, were selected to assess the effect of cultivation conditions on this ability.

Endophytic bacterial strains isolated from Ipomoea pes-caprae roots, TH10R, TH11T and TH13T are stored in the laboratory of the Department of Microbiological Biotechnology, Faculty of Biotechnology, Vietnam National Agricultural University.

The IAA concentration was determined by the method [3]. A calibration curve was constructed based on the IAA content in the test solution. IAA concentrations in solutions: $0 ; 10 ; 20 ; 30 ; 40 ; 50 ; 60 ; 70$ and $80 \mathrm{mg} / \mathrm{ml}$.

\subsection{Influence of cultivation time on IAA productivity and strain activity}

The IAA concentration was measured every $24 \mathrm{~h}$ in order to assess the influence of the cultivation time on the IAA-producing ability of the studied strains. Three selected endophytic strains were grown in a liquid nutrient medium Luria-Bertani containing peptone $10 \mathrm{~g} / \mathrm{l}$, yeast extract $5 \mathrm{~g} / \mathrm{l}, \mathrm{NaCl} 10 \mathrm{~g} / \mathrm{l}$, L-tryptophan $100 \mathrm{mg} / \mathrm{l}$, on a shaker at a rotation speed of $200 \mathrm{rpm}$. After every 24 hours of incubation, the culture fluid was centrifuged $(6000 \mathrm{rpm})$ for 10 minutes at $4{ }^{\circ} \mathrm{C}$. Then $1 \mathrm{ml}$ of the supernatant was vigorously stirred with $4 \mathrm{ml}$ of Salkovsky's reagent $\left(150 \mathrm{ml}\right.$ of concentrated $\mathrm{H}_{2} \mathrm{SO}_{4}, 250 \mathrm{ml}$ of distilled $\mathrm{H}_{2} \mathrm{O} ; 7.5 \mathrm{ml}$ of $0.5 \mathrm{M} \mathrm{FeCl}_{3} 6 \mathrm{H}_{2} \mathrm{O}$ ) and left in a stationary position for $20 \mathrm{~min}$ at room temperature before measuring on a spectrophotometer. Absorbance was determined at a wavelength of $530 \mathrm{~nm}$. The IAA concentration in each sample (option) was determined by comparison with a standard calibration curve.

\subsection{Influence of carbon and nitrogen sources on IAA productivity of the studied strains}

Three strains of endophyticbacteria were cultivated in Luria - Bertani liquid nutrient medium supplemented with $100 \mathrm{mg} / \mathrm{l} \mathrm{L-tryptophan.} \mathrm{As} \mathrm{various} \mathrm{sources} \mathrm{of} \mathrm{carbon} \mathrm{nutrition,}$ the following were selected: lactose, sucrose, sorbitol, xylose, glucose and starch at a concentration of $1 \%$. As various sources of nitrogen supply, the following were selected: $\left(\mathrm{NH}_{4}\right) \mathrm{H}_{2} \mathrm{PO}_{4}, \mathrm{NH}_{4} \mathrm{NO}_{3},\left(\mathrm{NH}_{4}\right)_{2} \mathrm{SO}_{4}, \mathrm{NH}_{4} \mathrm{Cl}, \mathrm{KNO}_{3}$ at a concentration of $5 \mathrm{~g} / \mathrm{l}$. 


\section{Results and discussion}

\subsection{Influence of cultivation time on IAA productivity of strains}

The dependence of the IAA amount synthesized by endophytic bacteria on the incubation time and the origin of the strains has been shown in the works of several researchers. Kumari et al. showed that the Bacillus subtilis DR2 strain accumulated the maximum IAA amount after $96 \mathrm{~h}$ of incubation [4]. Earlier, similar results were obtained by Bharucha et al. when they evaluated the effect of the cultivation time on the IAA-producing ability of the Pseudomonas putida UB1 strain [5]. Endophytic strain Klebsiella sp. RE3 from camellia roots produced the greatest IAA amount after 72 hours of cultivation [6]. Herlina et al. showed that several bacterial strains (BPK3, K1K1, AT, GNP2K1) isolated frompeanuts maximally synthesize IAA after $96 \mathrm{~h}$ of incubation, while other strains (GNP4K1, K1K2, GNP1K1) demonstrated the highest level of IAA production on the second cultivation day [7]. Bhutani et al. showed that for different Bacillusspp strains obtained from Vigna radiata tubercles, the maximum IAA concentration was observed in different periods (strain MBN3 - after $72 \mathrm{~h}$, strain MJHN1 - after $24 \mathrm{~h}$, strain MJHN10 - after $72 \mathrm{~h}$ of cultivation) [8].

In order to assess the influence of the cultivation time on IAA productivity, three selected strains were grown in liquid nutrient Luria - Bertani medium and the concentration of synthesized IAA in solution was measured after 48, 72, 96, and $120 \mathrm{~h}$ of incubation. The results are shown in Figure 1. It was found that after $48 \mathrm{~h}$ of cultivation, the IAA concentration of the studied strains ranges from $1.5 \mu \mathrm{g} / \mathrm{ml}$ in the TH13T strain to $3 \mu \mathrm{g} / \mathrm{ml}$ in the TH10R strain. The IAA concentration synthesized by three endophytic strains increases during the next $48 \mathrm{~h}$ and reaches a maximum at $96 \mathrm{~h}$ of cultivation. Further cultivation of the strains led to a decrease in IAA concentration in solution.

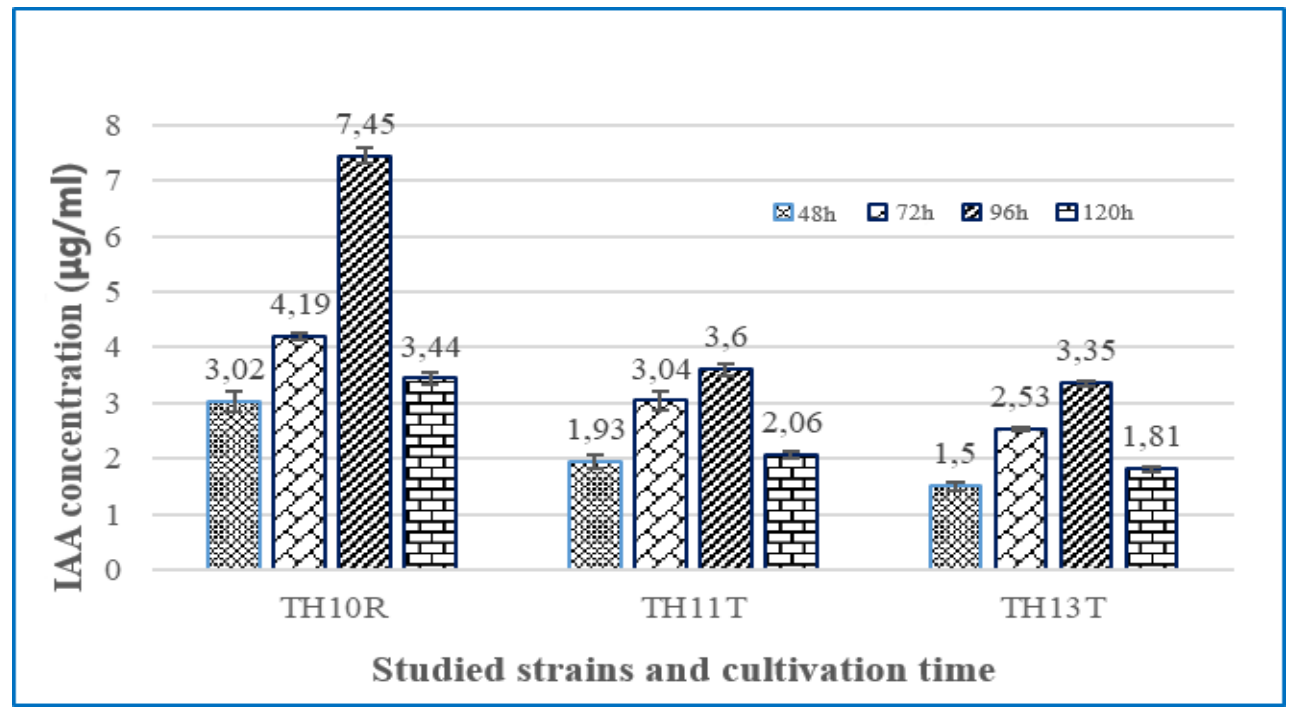

Fig. 1. Influence of cultivation time on IAA concentration.

In this work and those discussed above, it is noted that after reaching the maximum, the concentration of IAA begins to decrease. With further cultivation of bacterial strains, this indicator may decrease gradually or rather sharply. According to Patil et al. [9] and Arora et al. [10], this may be due to the appearance of IAA in test organisms, entering the stationary 
growth phase, aeration, adsorption of growth regulators on substrate particles, and the production of enzymes that decompose IAA, such as IAA oxidase and peroxidase, cultivation conditions, inaccessibility of substrates. The genetic factors influencing the level of IAA synthesis in bacteria can also be different. First, the genes' location for auxin biosynthesis in the genome modulates the level of IAA production [11]. Second, IAA production is associated with multiple pathways present in one bacterial species, and some bacteria possess more than one pathway that can be induced in different ways [12].

\subsection{Influence of carbon sources on IAA productivity of strains}

The carbon sources that are used by bacteria have a strong impact on the overall biosynthesis efficiency. It is known from previously published works that endophytic bacteria differ in the degree of carbon sources usage $[13,14]$. Glucose is reported to be the best carbon source for IAA production in Rhizobium spp. [15]. Sucrose is best suited to the Pantoea aglomerans and Pseudomonas putida UB1 strains, which, when used it, provided a relatively high IAA yield [5]. Nguyen Van Zhang et al. [6] showed that the Klebsiella sp. RE3 strain showed the highest IAA productivity when sorbitol was added. In an experiment by Kumari et al., Bacillus subtilis DR2 produced the maximum IAA amount when used in mannitol medium as a carbon source; sucrose was the next most effective carbon source [4]. In the experiments of Chandra et al., bacterial strains produced the greatest amount of IAA when dextrose was used in the medium [16]. Pseudomonas fluorescence in the experiment of Jeyanthi and Ganesh preferred lactose over other carbon sources during the growth and synthesis of IAA [17].

This study examined the effect of six different carbon sources (sorbitol, glucose, lactose, xylose starch, and sucrose) on IAA production (Fig. 2). It was experimentally established that the studied sources of carbon nutrition have different effects on the IAA productivity of the studied strains. Thus, a significant increase in IAA biosynthesis was obtained by cultivating the TH10R strain on a medium containing lactose and starch, the amount of IAA in this case amounted to 9.14 and $12.15 \mu \mathrm{g} / \mathrm{ml}$, respectively. When the TH11T strain is cultivated on a medium with a carbon source of glucose, xylose, or starch, the amount of IAA varies from 3.31 to $4.38 \mu \mathrm{g} / \mathrm{ml}$. Sucrose and sorbitol are not suitable for TH10R and TH11T strains, the amount of IAA produced by these strains is in the range from 1.11 to $1.69 \mu \mathrm{g} / \mathrm{ml}$ and 1.33 to $1.79 \mu \mathrm{g} / \mathrm{ml}$, respectively. Xylose, glucose, and sucrose are suitable for the TH13T strain, the amount of IAA in this case is in the range of 3.7 $5.91 \mu \mathrm{g} / \mathrm{ml}$; on a medium supplemented with sorbitol, this strain produces the lowest IAA concentration $(1.59 \mu \mathrm{g} / \mathrm{ml})$. 


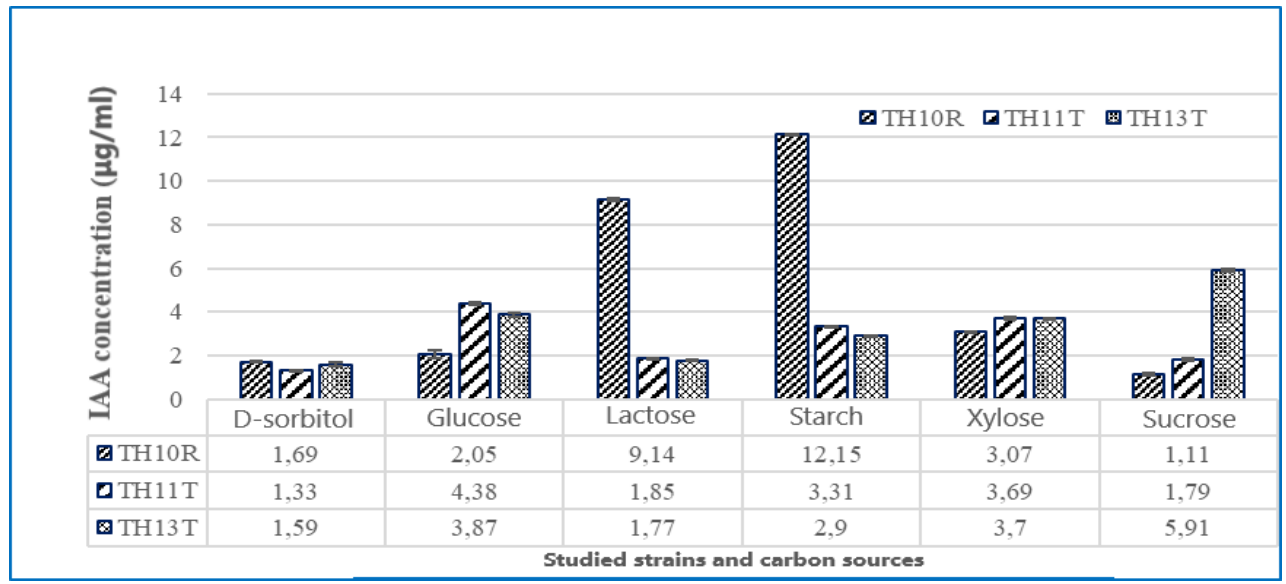

Fig. 2. Influence of carbon sources on IAA productivity of the studied strains.

\subsection{Influence of nitrogen sources on IAA productivity of strains}

The influence of nitrogen sources in the culture medium on IAA synthesis has been shown by many researchers $[18,19,20]$. Chandra et al. showed that strain CA2004 gives the maximum IAA amount when cultured on a medium supplemented with $\mathrm{NH}_{4} \mathrm{NO}_{3}$ and $\mathrm{KNO}_{3}$ [16]. In the study by Bharucha et al., the highest IAA concentration was produced by the Pseudomonas putida UB1 strain on a medium with $\left(\mathrm{NH}_{4}\right)_{2} \mathrm{SO}_{4}$, the next most efficient nitrogen sources were $\mathrm{KNO}_{3}$ and $\mathrm{NH}_{4} \mathrm{NO}_{3}$ [5]. The composition analysis of the nutrient medium revealed a stimulating effect of sorbitol and $\mathrm{NH}_{4} \mathrm{NO}_{3}$ on IAA productivity of the Klebsiella sp. RE3 strain [6].

The studied strains differed in the utilization degree of various nitrogen sources.

The TH10R strain synthesized the largest IAA amount on a medium supplemented with $\mathrm{NH}_{4} \mathrm{Cl}$ and $\mathrm{KNO}_{3}$, respectively, 9.55 and $8.26 \mu \mathrm{g} / \mathrm{ml}$, and the smallest - $1.63 \mu \mathrm{g} / \mathrm{ml}$ - with the addition of $\left(\mathrm{NH}_{4}\right)_{2} \mathrm{SO}_{4}$.

TH11T strain showed the maximum IAA concentration when $\mathrm{KNO}_{3}$ and $\mathrm{NH}_{4} \mathrm{NO}_{3}$ were added. The rest of the nitrogen sources are not suitable for this strain from the point of view of IAA production, the amount of which in the medium varied from 1.40 to $2.19 \mu \mathrm{g} / \mathrm{ml}$.

All the studied nitrogen sources had a negative effect on the IAA productivity of the TH13T strain. In a medium with these nitrogen sources, the TH13T strain drastically reduced the IAA production, the amount of which ranged from 1.12 to $1.93 \mu \mathrm{g} / \mathrm{ml}$. 


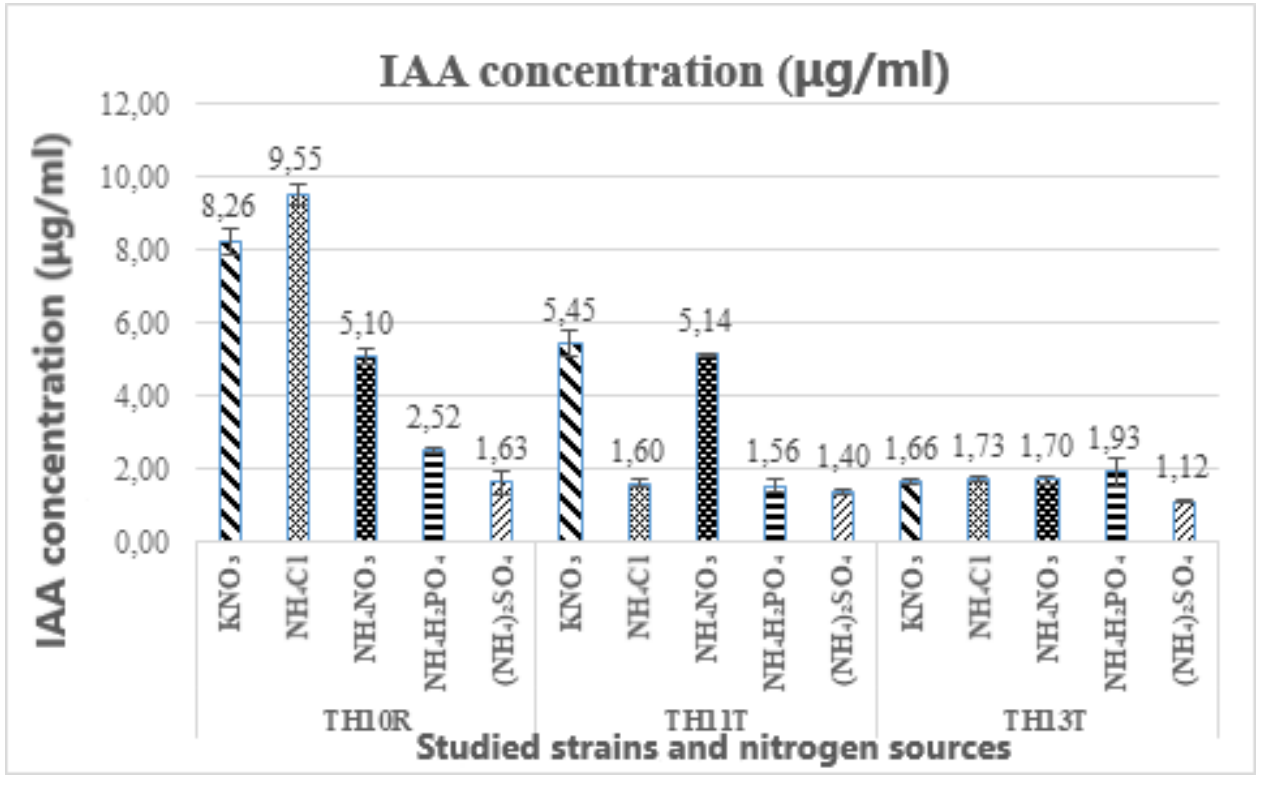

Fig. 3. Influence of various nitrogen sources on IAA productivity of the studied strains.

\subsection{Molecular identification of TH10R strain}

Of the three strains studied, the TH10R strain, which produced the maximum IAA amount, was sent to the Laboratory of Molecular Biology and Applied Biotechnology of the Faculty of Biotechnology of the Vietnam National Agricultural University for its nucleotide sequence of the 16Sr RNA gene and subsequent species identification based on this trait. The analysis result of the 16S rRNA nucleotide sequence of the TH10R strain using the NCBI BLAST program showed a close relationship of the studied strain with the Bacillus mycoides strains (Fig. 4) and, on this basis, was named Bacillus mycoides TH10R.

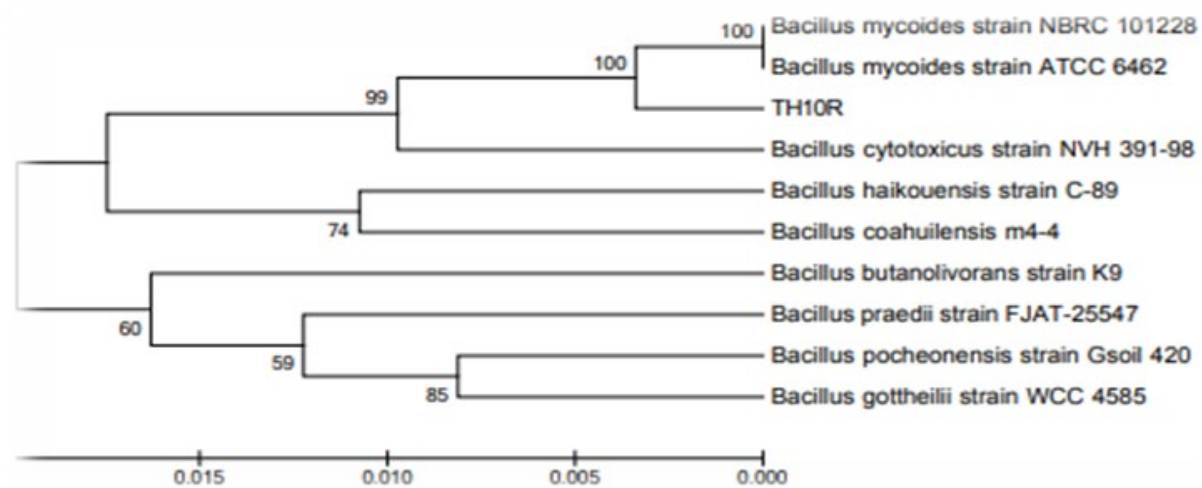

Fig. 4. Phylogenetic analysis of 16Sr RNA strain TH10R. 


\section{Conclusions}

The study results of the cultivation conditions influence on IAA-producing ability of three strains of endophytic bacteria from the roots of Ipomoea pes-caprae TH10R, TH11T, TH13T allow to conclude that all three strains accumulate the maximum amount of IAA during $96 \mathrm{~h}$ of cultivation. The TH11T strain synthesized the maximum IAA amount when glucose or xylose was added to the nutrient medium as a carbon source and $\mathrm{KNO}_{3}$ or $\mathrm{NH}_{4} \mathrm{NO}_{3}$ as a nitrogen source. Sucrose is the most suitable carbon source for the TH13T strain. All studied nitrogen sources negatively affected the ability to synthesize IAA in the TH13T strain. A significant increase in IAA biosynthesis was observed when the TH10R strain was cultivated in a medium containing lactose or starch as the carbon source and $\mathrm{NH}_{4} \mathrm{Cl}$ or $\mathrm{KNO}_{3}$ as the nitrogen source. The TH10R strain, which produced the maximum amount of IAA out of the three studied strains, was named Bacillus mycoides TH10R based on the results of molecular identification.

\section{References}

1. E.A.Leontievskaya, Structure of epiphytic-saprotrophic bacterial complexes of grain and vegetable crops (2014)

2. I.V. Maksimov, Modern approaches and methods in plant protection (2018)

3. E. Glickmann, Y. Dessaux Appl. Environ. Microbiol. 61 (1995)

4. S. Kumari, C. Prabha, A. Singh et al. International Journal of Pharma Medicine and Biological Sciences. 7(2) (2018)

5. U. Bharucha, K. Patel, U.B. Trivedi Agric Res 2 (2013)

6. Nguyen Van Zhang, Wu Thi Ngoc Ziep, Pham Han Huen, Kalashnikova E.A., Kirakosyan R.N. Questions of biological, medical and pharmaceutical chemistry23 (11) 2020

7. L. Herlina, K.K. Pukan, D. Mustikaningtyas, Cell Bio Dev.,1 (2017)

8. N. Bhutani, R. Maheshwari, R. Negi, P. Suneja, Israel journal of plant sciences $\mathbf{6 5}$ (2018)

9. N. B. Patil, M. Gajbhiye, S. S. Ahiwale, A. B. Gunjal, B. P. Kapadnis, Int. J. Envir. Sci. 1 (2011)

10. P.K. Arora, A. Sharma, H. Bae J. Chem. (2015) [Online] Available: http://dx.doi.org/10.1155/2015/129159

11. C.L. Patten, B.R. Glick, Can. J. Microbiol. 42 (1996)

12. S. Manulis, A. Haviv-Chesner, M.T. Brandl, S.E. Lindow, I. Barash, Mol. Plant Microbe Interact. 11(7) (1998)

13. B. Mohite J. Soil Sci. Plant Nut. 13 (2013)

14. A. Kumar, R. Singh, A. Yadav, D.D. Giri, P.K. Singh, K.D. Pandey, 3 Biotech 6(1) (2016)

15. P.S. Basu, A.C. Ghosh, Acta Biotechnol. 21 (2001)

16. S. Chandra, A. Kazim, K. Madhumita, Journal of Genetic Engineering and Biotechnology 16 (2018)

17. V. Jeyanthi, P. Ganesh, Int J. Pharma Biol Arch.4(2) (2013)

18. C. Leelahawonge, N. PongsilpThamasat, Int J Sci Tech.14(2) (2009) 
19. M. Santi, C. Keshab, S. Dey, B.R. Pati, Res J Microbiol.,2 (2007)

20. M. Sridevi, N.C.S. Yadav, K.V. Mallaiah, Res J. Microbiol.3(4) (2008) 